übte, beleuchtete Aron in Derfalsche Messias. ${ }^{370}$ In seinem letzten Beitrag für den Monat mit dem Titel Noch einmal: Hitler. Wie haltbar ist die Totalitarismus-Theorie untersuchte Aron den Nationalsozialismus unter explizit totalitarismustheoretischer Perspektive und verglich in diesem Zusammenhang die Verlaufsform der »roten und braunen Revolution ${ }^{371}$

Aron hat nach Kriegsende ein umfangreiches, facettenreiches Gesamtwerk hinterlassen, das neben dem Nachdenken über das Totalitarismusphänomen unter anderem die Themenbereiche Soziologie, ${ }^{372}$ internationale Beziehungen, ${ }^{373}$ Ideologiekritik, ${ }^{374}$ (Welt-)Politik ${ }^{375}$ und politische Theorie umfasste. Unter dem Einfluss von Tocqueville stehend, bestand das primäre Ziel von Arons Reflexionen in der Legitimation von politischer Freiheit. ${ }^{376}$ Neben verschiedenen Lehrtätigkeiten ab 1945 - z. B. als Soziologieprofessor an der Pariser Sorbonne - war Aron journalistisch tätig. Von 1947 bis 1977 schrieb er im Figaro wöchentlich Kommentare zur internationalen Politik und fungierte danach bis zu seinem Tode im Oktober 1983 als Herausgeber und Kolumnist für das Wochenmagazin L'Express.

\title{
4.7 Hans Kohn
}

$\mathrm{Zu}$ einem der wichtigsten Autoren des Monat entwickelte sich der jüdische Historiker und Nationalismusforscher Hans $\mathrm{Kohn}^{377}$, der sich nicht zuletzt aufgrund seiner individuellen Erfahrungen in seinen zahlreichen Veröffentlichungen mit den ideen- und gesellschaftsgeschichtlichen Ursprüngen der politischen und geistigen Erschütterun-

370 Der falsche Messias. Ein Schlußwort zu »The God that failed«, in: Der Monat 3 (1950), H. 26, S. 175-184 (hierbei handelte es sich um das Nachwort für die französische Ausgabe von Richard H. S. Crossmanns Sammelband The God that failed, das unter dem Titel Le Dieu des Ténèbres 1950 in Paris erschien).

371 Noch einmal: Hitler. Wie haltbar ist die Totalitarismus-Theorie?, in: Der Monat 278 (1981), H. 1, S. 4255. Eine längere Fassung erschien unter dem Titel Gibtes ein Nazi-Rätsel in: Stark, Raymond Aron und der Gestaltwandel des Totalitarismus, S. 294-320.

372 Siehe u. a.: La societé industrielle et la guerre, Paris 1958 sowie Dix-huit leçons sur la societé, Paris 1962 (dt. Ausg.: Die industrielle Gesellschaft, Frankfurt a. M. 1964)

373 Siehe u. a.: Paix et guerre entre les Nations, Paris 1961 (dt. Ausg.: Frieden und Krieg. Eine Theorie der Staatenwelt, Frankfurt a. M. 1963).

374 Siehe u. a.: Marxismes imaginaires. D’une famille à l'autre, Paris 1970 (dt. Ausg.: Die heiligen Familien des Marxismus, Hamburg 1970) sowie Les Dèsillusions du progrès, Paris 1969 (dt. Ausg. Fortschritt ohne Ende. Über die Zukunft der Industriegesellschaft, München 1970).

375 Siehe u. a.: La tragédie algérienne, Paris 1957; L’Algérie et la République, Paris 1958; République impériale, les Etats-Unis dans le monde 1945-1972, Paris 1973 (dt. Ausg.: Die imperiale Republik: die Vereinigten Staaten von Amerika und die übrige Welt seit 1945, Stuttgart 1975); Plaidoyer pour l'Europe dècadente, Paris 1977 (dt. Ausg.: Plädoyer für das dekadente Europa); La Révolution introuvable. Réflexions sur la Révolution de Mai, Paris 1968.

376 Siehe hierzu bes.: Essai sur les libertés, Paris 1965 (dt. Ausg.: Über die Freiheiten. Essay, Stuttgart 1981) sowie Démocratie et totalitarisme, Paris 1965 (dt. Ausg.: Demokratie und Totalitarismus, Hamburg 1970).

377 Bevor Kohn als Autor für den Monat in Erscheinung trat, veröffentlichte er - wie auch beispielsweise Arendt - bereits in der ab 1945 (bis 1949) von Dolf Sternberger - unter Mitwirkung von Karl Jaspers, Werner Kraus und Alfred Weber-herausgegebenen Monatszeitschrift Die Wandlung. 
gen des 20. Jahrhunderts intensiv auseinandersetzte. Zudem zielten seine Reflexionen in der Zeitschrift auf die sich zwingend ergebenden grundsätzlichen politischen Folgen nach dem Ende des Zweiten Weltkrieges - wie zum Beispiel die Bedeutung der Menschenrechte und die Rolle der Vereinten Nationen. ${ }^{378}$

Kohn wurde am 15. September 1891 in Prag geboren und studierte an der dortigen Universität Rechts- und Staatswissenschaften. Der Vater war Geschäftsmann. Die aus einer wohlhabenden Familie stammende, kulturell sehr gebildete Mutter sorgte für eine mehrsprachige Erziehung ihres Sohnes, wie es Kohn in seiner Autobiografie hervorhob. ${ }^{379}$ Bereits als junger Student beschäftigte sich Kohn intensiv mit der Geschichte und dem seit der Jahrhundertwende ausbreitenden Nationalismus, da der »Bürger vieler Welten« miterleben musste, wie seine Heimat, der Vielvölkerstaat der österreichischen Monarchie, in der »steigenden Flut des Nationalismus « unterging. ${ }^{380}$ An der Prager Universität exponierte er sich in der jüdischen Studentenvereinigung Barkochba und bezog schon zu diesem Zeitpunkt Position gegen nationalistische auch zionistische - Selbstüberschätzung. ${ }^{381}$ Kohn sympathisierte unter dem Einfluss der Ideen Gustav Landauers eher zum anti-autoritären Anarchosozialismus. ${ }^{382}$

Der 1914 ausgebrochene Erste Weltkrieg und seine Folgen zeigten Kohn die dünne Firnis der Zivilisation. Ebendiese »Urkatastrophe« des 20. Jahrhunderts wurde zum zentralen Ausgangspunkt für seine späteren wissenschaftlichen Veröffentlichungen sowie zur eigentlichen Schockerfahrung, denn, so Kohn, »dieser Konflikt veränderte fast über alles Begreifen die Ordnung und Aussichten meines persönlichen Lebens, meines Heimatlandes, der Völker Europas und der Völker aller andern Erdteile. Der Krieg war der Auftakt der ersten weltweiten Revolution«, d.h. der Russischen Revolution von 1917. ${ }^{383}$ Den Verlauf der bolschewistischen Revolution erlebte Kohn konkret mit, da er zu Beginn des Ersten Weltkrieges in russische Kriegsgefangenschaft geriet, die dazu führte, dass er fast fünf Jahre in Russland verbringen musste. Nach seiner Freilassung aus der sibirischen Gefangenschaft machte er eine lange Reise durch das Land, die es ihm ermöglichte, seine russischen Sprachkenntnisse zu vertiefen und sowohl die Menschen als auch die Kultur kennenzulernen. Als Ergebnis des Studiums der russischen Geschichte entstanden 1923 die Untersuchungen Sinn und Schicksal der

378 Siehe zum Beispiel: Das Jahrhundert des Verrats, in: Der Monat 1 (1948), H. 3, S. 7-13 (Auszug von: Das Zwanzigste Jahrhundert. Eine Zwischenbilanz des Westens, Zürich/Wien/Konstanz 1950); Gegner der Welteinheit, in: Der Monat 1 (1948), H. 7, S. 19-24; Dostojewski. Ein politisches Portrait, in: Der Monat 2 (1949), H. 13, S. 78-88, sowie H. 14, S. 187-192 (Auszug von: Propheten ihrer Völker. Mill, Michelet, Treitschke, Dostojewski: Studien zur Ceschichte des europäischen Nationalismus im 19. Jahrhundert, Bern 1949); Ein Vorspiel zum totalitären Terror, in: Der Monat 3 (1951), H. 35, S. 512-518; Rußland vor Lenin, in: Der Monat 6 (1954), H. 67, S. 75 f. (Rezension); Toynbees Claubensbekenntnis, in: Der Monat 7 (1955), H. 83, S. 465-469 (Rezension).

379 Hans Kohn, Bürger vieler Welten. Ein Leben im Zeitalter der Weltrevolution, Frauenfeld 1965, S. 55.

380 Ebd., S. 28.

381 In einem Artikel für die Zeitschrift Die Juden kritisierte Kohn bereits 1919 die Juden Palästinas in Bezug auf ihre chauvinistische Einstellung gegenüber den Arabern. Siehe Young-Bruehl, Hannah Arendt-Leben, Werk und Zeit, S. 319.

382 Vgl. Hans Kohn/Hugo Bergmann, In Memory of Gustav Landauer, Tel Aviv 1929.

383 Kohn, Bürger vieler Welten, S. 15. 
Revolution ${ }^{384}$ sowie - nach einer neuerlichen Reise durch die Sowjetunion im Sommer 1932 - die Studie Der Nationalismus in der Sowjetunion ${ }^{385}$.

Bei einem kurzen Aufenthalt in Berlin im Januar 1920 nahm Kohn einen extremen aggressiven Nationalismus nach der deutschen Niederlage im Ersten Weltkrieg in der Bevölkerung wahr. Er kehrte 1920 nach Prag zurück und promovierte dort 1923 zum Dr. jur. Anschließend lebte er in Paris und London und ab 1925 bis 1931 in Jerusalem. In diesen Jahren entstanden nach intensiver Forschung über die Geschichte des Nationalismus und Kolonialismus im Mittleren Osten sowie Reisen durch die arabischen Länder unter anderem die Bücher Eine Geschichte der arabischen nationalen Bewegung ${ }^{386}$, Geschichte der nationalen Bewegung im Orient ${ }^{387}$ und schließlich Nationalismus und Imperialismus im Vorderen Orient ${ }^{388}$. Des Weiteren erschienen mehrere Arbeiten zum Thema Judentum und Zionismus: Nationalismus, über die Bedeutung des Nationalismus im Judentum und in der Gegenwart ${ }^{389}$, Die politische Idee des Judentums ${ }^{390}$, Zionistische Politik ${ }^{391}$, Über die Geschichte der zionistischen Idee ${ }^{392}$ und die Biografie Martin Buber. Sein Werk und seine Zeit. ${ }^{393}$ Diese historischen Forschungen und der sechs Jahre lange Aufenthalt in Palästina machten Kohn sensibel für den sich anbahnenden Konflikt zwischen Juden und Arabern um den »heiligen Ort« Palästina. Von 1925 bis 1933 fungierte er für die Frankfurter Zeitung sowie die Neue Zürcher Zeitung als Korrespondent für den Mittleren Osten.

In seiner Autobiografie berichtete Kohn hinsichtlich der NZZ, dass er im Verlauf der 1930er-Jahre mit großer Sympathie den Kampf der renommierten Schweizer Tageszeitung zur »Verteidigung der westlichen Freiheit« gegen den Faschismus und den Nationalsozialismus verfolgte. In dieser Zeit entstand auch eine lebenslange Freundschaft mit dem Redakteur der NZZ und später sehr bekannten Philosophen Hans Barth. $^{394}$

Eine Vortragsreise zu Beginn der 1930er-Jahre brachte Kohn über Europa Ende 1931 in die Vereinigten Staaten. Nach seinem Entschluss, sich endgültig in den USA niederzulassen, nahm er - nach erfolgreichem Unterricht an der führenden Hochschule für höhere Erwachsenenbildung, der New School for Social Research in New York - als Professor für Neuere Geschichte Lehrtätigkeiten an mehreren Colleges und Universitäten auf. 1935 hielt er auf der in Minneapolis veranstalteten Konferenz »Diktatur in der modernen Welt« einen Vortrag, auf der er unter anderem auf den neuartigen Cha-

384 Hans Kohn, Sinn und Schicksal der Revolution, Wien 1923.

385 Ders., Der Nationalismus in der Sowjetunion, Frankfurt a. M. 1932. Dieser Band entstand schlussendlich aufgrund einer im Auftrag der Frankfurter Zeitung gemachten Reise in die Sowjetunion im Laufe des Sommers 1931, um das Nationalitätenproblem zu studieren.

386 Ders., Eine Geschichte der arabischen nationalen Bewegung, Tel Aviv 1926.

387 Ders., Geschichte der nationalen Bewegung im Orient, Berlin 1928.

388 Ders., Nationalismus und Imperialismus im Vorderen Orient, Frankfurt a. M. 1931.

389 Ders., Nationalismus. Über die Bedeutung des Nationalismus im Judentum und in der Gegenwart, Wien 1922.

390 Ders., Die politische Idee des Judentums, München 1924.

391 Ders., Robert Weltsch, Zionistische Politik, Mährisch-Ostrau 1927.

392 Hans Kohn, Über die Geschichte der zionistischen Idee, 2 Bde., Warschau 1929 und 1930.

393 Ders., Martin Buber. Sein Werk und seine Zeit. Ein Versuch über Religion und Politik, Hellerau 1930.

394 Ders., Bürger vieler Welten, S. 190. 
rakter der "totalen Staaten« in Deutschland, Italien und Russland als eine Folge des Ersten Weltkrieges insistierte und zudem die Rolle des Nationalismus beleuchtete. ${ }^{395}$

Nach dem Ausbruch des Zweiten Weltkrieges engagierte sich Kohn auf unterschiedlichen Ebenen gegen den Nationalsozialismus. Neben seinen Seminaren an der Harvard Universität - die unter anderem von den später sehr bekannten Wissenschaftlern Karl Deutsch und Peter Viereck besucht wurden - hielt er beispielsweise in jenen kritischen Jahren wöchentlich öffentliche Vorlesungen (zeitweise mit Max Lerner) über die internationale Lage. Auf der unter dem Eindruck des 1939 abgeschlossenen deutsch-sowjetischen Nichtangriffspaktes stattgefundenen Totalitarismuskonferenz im November 1939 in Philadelphia setzte sich Kohn in seinem Vortrag The Totalitarian Philosophy of War explizit mit dem Nationalsozialismus auseinander. ${ }^{396}$ In diesen Jahren arbeitete er zudem intensiv an seinem 1944 erschienenen und zum Standardwerk avancierten Buch The Idea of Nationalism. A Study in its Origins and Background ${ }^{397}$, das ausdrücklich an die Forschungsarbeiten aus den 1920er-Jahren anknüpfte. Der Nationalismus hatte ihn seit diesem frühen Zeitpunkt als ein geschichtlich-politisches Problem »in seinen Bann gezogen«, teilte der Autor in seinem Vorwort mit. ${ }^{398}$ Eingedenk der fatalen Rolle, die der Nationalismus im Ersten Weltkrieg und im Faschismus, im Nationalsozialismus sowie im Sowjetkommunismus gespielt hatte, untersuchte Kohn in seiner beinahe 1000 Seiten umfassenden Studie die Entwicklung des Nationalismus vom Altertum bis zur Epoche der Aufklärung und den Revolutionen in Amerika und Frankreich. Das Buch war als ein Band in einer Reihe geplant, die den Titel Das Zeitalter des Nationalismus tragen sollte, indes letzten Endes nicht realisiert wurde. ${ }^{399}$

Mit der Verwundbarkeit der westlichen Zivilisation angesichts von zwei Weltkriegen und den »totalitären Revolutionen« des Faschismus, des Nationalsozialismus und

395 Ders, Communist and Fascist Dictatorship. A Comparative Study, in: Ford, Dictatorship in the Modern World, S. 141-160; deutsche Übersetzung abgedruckt in: Seidel/Jenkner, Wege der Totalitarismusforschung, S. 49-63, vgl. hierzu auch Kohn, Revolutions and Dictatorships. Siehe auch die entsprechenden Ausführungen im Einleitungskapitel.

396 Hans Kohn, The Totalitarian Philosophy of War, in: Symposium of the Totalitarian State. From the Standpoints of History, Economics and Sociology, Nov. 17, 1939. Proceedings of the American Philosophical Society, Bd. 82 (1940), Nr. 1, Philadelphia 1940, S. 57-72. Siehe auch die entsprechenden Ausführungen im Einleitungskapitel.

397 Ders., The Idea of Nationalism. A Study in its Origins and Background, New York 1944 (dt. Ausg.: Die Idee des Nationalismus. Ursprung und Geschichte bis zur Französischen Revolution, Heidelberg 1950).

398 Ders., Die Idee des Nationalismus, S. 10.

399 Teilstücke dieser geplanten Reihe mit dem Titel Das Zeitalter des Nationalismus wurden als einzelne Bücher veröffentlicht: Prophets and Peoples: Studies in Nineteenth-Century Nationalism, New York 1946 (dt. Ausg.: Propheten ihrer Völker. Mill, Michelet, Treitschke, Dostojewski: Studien zur Geschichte des europäischen Nationalismus im 19. Jahrhundert, Bern 1949); Pan-Slavism, Its History and Ideology, University of Notre Dame Press 1953 (dt. Ausg.: Die Slawen und der Westen. Die Geschichte des Panslawismus, Wien 1956); Der schweizerische Nationalgedanke, Eine Studie zum Thema Nationalismus und Freiheit, Zürich 1955; American Nationalism. An Interpretative Essay, New York 1957; The Mind of Cermany, New York 1960 (dt. Ausg.: Wege und Irrwege. Zur Geistesgeschichte des bürgerlichen Deutschland, Düsseldorf 1962); The Age of Nationalism: The First Era of Clobal History, New York 1962; vgl. auch Hans Kohn, Nationalism, its Meaning and History, Princeton 1955 (dt. Ausg.: Von Machiavelli zu Nehru. Zur Problemgeschichte des Nationalismus, Freiburg i. Br. 1964). 
des Sowjetkommunismus beschäftigte sich Kohn in dem Band The Twentieth Century. A Mid-way Account of the Western World. ${ }^{400}$

In den Vereinigten Staaten unterstützte Kohn im Anschluss an das Ende des Zweiten Weltkrieges - neben Martin Buber, Erich Fromm, James Marshall, Maurice Hexter, David Riesman, Hannah Arendt und dem Herausgeber der Zeitschrift Commentary, Elliot Cohen - die jüdische Organisation Ihud von Judah Magnes und engagierte sich politisch in Bezug auf die Gründung des Staates Israel. In diesem Kontext befürwortete er eine arabisch-israelische, d. h. binationale Lösung des Palästinaproblems.

Neben den bereits angeführten diversen Veröffentlichungen trat Kohn in den 1950er- und 1960er-Jahren zudem als Autor von zahlreichen weiteren Büchern in Erscheinung. ${ }^{401} \mathrm{Zu}$ seinen Freunden in den Vereinigten Staaten gehörte unter anderem der Totalitarismusforscher Sigmund Neumann. ${ }^{402}$ Zuletzt lebte Kohn in Philadelphia, wo er am 16. März 1971 verstarb.

\subsection{Hannah Arendt}

Die Schriften Hannah Arendts wurden fast sämtlich unter dem Eindruck der übermächtigen Destruktionsgeschichte des 20. Jahrhunderts angefertigt. Aufgrund der existenziellen Erfahrung von Verfolgung, des Flüchtlings, der Asylsuchenden, Internierten und Staatenlosen, von Gewalt und Terror - sprich einer paradigmatischen Grunderfahrung des letzten Jahrhunderts - kam es bei ihr ausdrücklich zu einer Verflechtung von Werk und Biografie. Die mit ihrer 1951 in den Vereinigten Staaten erschienenen Untersuchung The Origins of Totalitarianism ${ }^{403}$ international bekannt gewordene Arendt gehörte frühzeitig zu den Autorinnen des Monat. ${ }^{404}$ Neben einem literarischen Porträt Hermann Brochs ${ }^{405}$ und einem Rezensionsaufsatz zur Geschichte des >Dritten Reiches` mit dem Titel Bei Hitler zu Tisch ${ }^{406}$ standen drei Auszüge ihrer

400 Ders., The Twentieth Century. A Mid-way Account of the Western World, New York 1949 (dt. Ausg.: Das Zwanzigste Jahrhundert. Eine Zwischenbilanz des Westens, Zürich/Wien/Konstanz 1950); siehe hierzu Joachim G. Leithäuser, Unbarmherzige Ratio, in: Der Monat 3 (1951), H. 30, S. 655-657 (Rezension). Siehe hierzu auch Kap. II.1.

401 Siehe exemplarisch Hans Kohn, Is the liberal west in decline, London 1957 (dt. Ausg.: Ist die freie Welt zum Untergang verurteilt?, Köln 1959).

402 Neumann, Permanent Revolution. Kohn zeichnete für die nach Neumanns Tod im Jahre 1962 herausgegebene zweite Auflage verantwortlich. Diesen Hinweis entnahm ich dem Aufsatz von Söllner, Sigmund Neumanns »Permanent Revolution«, S. 53, Anm. 2.

403 Arendt, The Origins of Totalitarianism. Zu den aus Anlass des fünfzigsten Erscheinungsjahres der Studie veranstalteten Tagungen an der Berlin-Brandenburgischen Akademie der Wissenschaft in Berlin und an der Carl von Ossietzky Universität Oldenburg im Dezember 2001 siehe Antonia Crunenberg (Hg.), Totalitäre Herrschaft und republikanische Demokratie. Fünfzig Jahre The Origins of Totalitarianism von Hannah Arendt, Frankfurt a. M. 2003.

404 Vor der Mitarbeit für den Monat veröffentlichte Arendt - wie auch beispielsweise Kohn - bereits in der von ihren Freunden Dolf Sternberger und Karl Jaspers ab 1945 (bis 1949) (mit-)herausgegebenen Zeitschrift Die Wandlung. Sie publizierte dort die Beiträge Franz Kafka, von neuem gewürdigt, Organisierte Schuld, Über den Imperialismus, Konzentrationsläger, Es gibt nur ein einziges Menschenrecht sowie Parteien und Bewegungen.

405 Hermann Broch und der moderne Roman, in: Der Monat 1 (1949), H. 8/9, S. 147-151.

406 Bei Hitler zu Tisch, in: Der Monat 4 (1951), H. 37, S. 85-90 (Rezension). 